\title{
9 GEOMETRY AND TOPOLOGY OF POLYGONAL LINKAGES
}

\author{
Robert Connelly and Erik D. Demaine
}

\section{INTRODUCTION}

There is a long and involved history of linkages starting at least in the nineteenth century with the advent of very complicated and intricate machinery. Some of the practical problems involved led to interesting, nontrivial geometric problems, and even recently there has been progress on some very basic questions. We will attempt to point the reader to some of the results that we know in this direction.

There are several points of view and groups of people working on various aspects of the theory of linkages, but they seem to be disjointed, with each group unaware of other groups that are in related or even overlapping fields. Despite that, we will also try to point out connections when we can.

\subsection{MATHEMATICAL THEORY OF LINKAGES}

The underlying principles and definitions are mathematical and in particular geometric. Despite the long history of kinematics, even theoretical kinematics (see, e.g., Bottema and Roth [BR79a]), only since the 1970s does there seem to be any systematic attempt to explore the mathematical and geometric foundations of a theory of linkages.

We begin with some definitions, some of which follow those in rigidity theory described in Chapter 60. The rough, intuitive notions are as follows. A linkage is a combinatorial structure plus edge lengths, and we often distinguish three special types of linkages: arcs, cycles, and trees. A configuration realizes a linkage in Euclidean space, a reconfiguration (or flex) is a continuum of such configurations, and the configuration space embodies all reconfigurations. The configuration space can be considered either allowing or disallowing bars to intersect each other.

\section{GLOSSARY}

Bar linkage or linkage: A graph $G=(V, E)$ and an assignment $\ell: E \rightarrow \mathbb{R}^{+}$of positive real lengths to edges.

Vertex or joint: A vertex of a linkage.

Bar or link: An edge $e$ of a linkage, which has a specified fixed length $\ell(e)$.

Polygonal arc: A linkage whose underlying graph is a single path. (Also called 
FIGURE 9.1.1

Different types of linkages, according to whether the underlying graph is a path, cycle, or tree, or the graph is arbitrary.

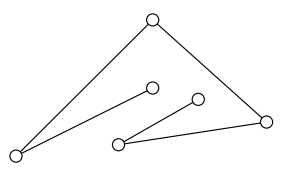

arc / open chain

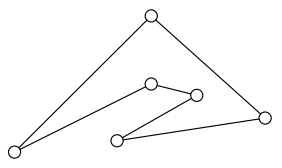

cycle / closed chain

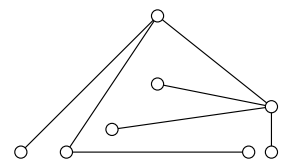

tree

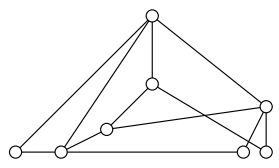

general

\section{FIGURE 9.1.2}

Snapshots of a reconfiguration of a polygonal arc.

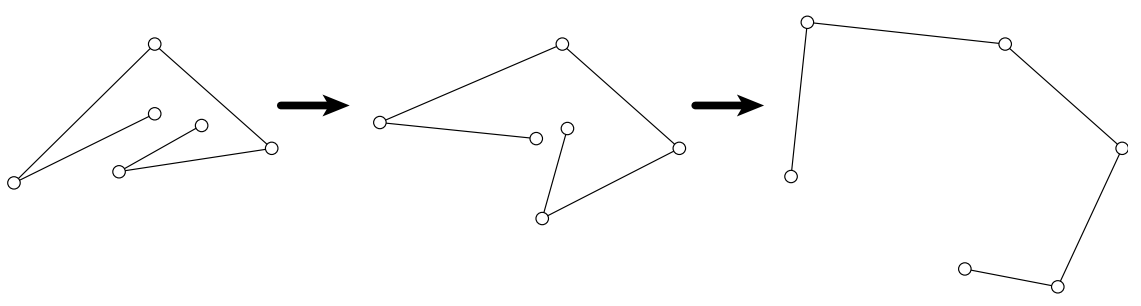

an open chain or a ruler.)

Polygonal cycle: A linkage whose underlying graph is a single cycle. (Also called a closed chain or a polygon.)

Polygonal tree: A linkage whose underlying graph is a single tree.

Configuration of a linkage in d-space: A mapping $p: V \rightarrow \mathbb{R}^{d}$ specifying a point $p(v) \in \mathbb{R}^{d}$ for each vertex $v$ of the linkage, such that each bar $\{v, w\} \in E$ has the desired length $\ell(e)$, i.e., $|p(v)-p(w)|=\ell(e)$.

A configuration can be viewed as a point $p$ in $\mathbb{R}^{d|V|}$ by arbitrarily ordering the vertices in $V$, and assigning the coordinates of the $i$ th vertex $(0 \leq i<|V|)$ to coordinates $i d+1, i d+2, \ldots, i d+d$ of $p$.

Framework or bar framework: A linkage together with a configuration.

Reconfiguration or motion or flex of a linkage: A continuous function $f$ : $[0,1] \rightarrow \mathbb{R}^{d|V|}$ specifying a configuration of the linkage for every moment in time between 0 and 1 .

Configuration space or Moduli space of a linkage: The set $\mathcal{M}$ of all configurations (treated as points in $\mathbb{R}^{d|V|}$ ) of the linkage.

Self-intersecting configuration: A configuration in which two bars intersect but are not incident in the underlying graph of the linkage.

Reconfiguration avoiding self-intersection: A reconfiguration $f$ in which every configuration $f(t)$ does not self-intersect.

Configuration space of a linkage, disallowing self-intersection: The subset $\mathcal{F}$ of the configuration space $\mathcal{M}$ in which every configuration does not selfintersect. (Also called the free space of the linkage.) 
Paths in the configuration space of a linkage capture the key notion of reconfiguration (either allowing or disallowing self-intersection as appropriate). Many important questions about linkages can be most easily phrased in terms of the configuration space. For example, we are often interested in whether the configuration space is connected (every configuration can be reconfigured into every other configuration), or in the topology of the configuration space.

\subsection{CONFIGURATION SPACES OF ARCS AND CYCLES WITH POSSIBLE INTERSECTIONS}

One fundamental problem is to compute the topology of the configuration space of planar polygonal cycles (polygons), allowing possible self-intersections. There is a long list of results in increasing generality for computing information about the algebraic topological invariants of this configuration space. One approach is Morse Theory, which reveals some of the basic information, in particular, the connectivity and some of the easier invariants such as the Euler characteristic.

\section{CONNECTIVITY}

The following is an early result possibly first due to [Hau91], but rediscovered by [Jag92], and then rediscovered again or generalized considerably by many others, in particular, [Kam99, KT99, MS00, KM95, LW95].

THEOREM 9.2.1 Connectivity for planar polygons [Hau91]

Let $s_{1} \leq s_{2} \leq \cdots \leq s_{n}$ be the cyclic sequence of bar lengths in a polygon, and let $s=s_{1}+s_{2}+\cdots+s_{n}$. Then

i) The configuration space is nonempty if and only if $s_{n} \leq s / 2$.

ii) The configuration space, modulo orientation-preserving congruences, is connected if and only if $s_{n-2}+s_{n-1} \leq s / 2$. If the space is not connected, there are exactly two connected components, where each configuration in one component is the reflection of a configuration in the other component.

The configuration space is a smooth manifold if and only if there is some configuration $p$ with all its vertices on a line, which in turn is determined by the edge lengths as described above. Also, the configuration space remains congruent no matter how we permute the cyclic sequence of bar lengths. When the linkage is not allowed to self-intersect, it is common to consider the configuration space modulo all congruences of the plane (including reflections); but when self-intersections are allowed, and condition ii) above is satisfied, it is possible to move the linkage from any configuration to its mirror image.

For polygons in dimensions higher than two, the situation is simpler:

THEOREM 9.2.2 Connectivity for nonplanar polygons [LW95]

The configuration space of a polygon in d-dimensional space, for $d>2$, is always connected. 


\section{HOMOLOGY, COHOMOLOGY, AND HOMOTOPY}

After connectivity, there remains the calculation of the higher homology groups, cohomology groups, and the homotopy type of the configuration space. Here is one special case as an example:

THEOREM 9.2.3 Configuration space of equilateral polygons [KT99]

Let $\mathcal{M}$ be the configuration space of a polygon with $n$ equal bar lengths, modulo congruences of the plane. The homology of $\mathcal{M}$ is a torsion-free module given explicitly in [KT99]. When $n$ is odd, $\mathcal{M}$ is a smooth manifold; and when $n=5, \mathcal{M}$ is the compact, orientable two-dimensional manifold of genus 4 (originally shown in [Hav91], as well as in [Jag92]).

See also especially [KM95] for some of the basic techniques. For calculating the configuration space of graphs other than a polygon, see in particular the article [TW84], where a particular linkage, with some pinned vertices, has a configuration space that is an orientable two-dimensional manifold of genus 6 .

Another case that has been considered is an equilateral polygon in 3-space with angles between incident edges fixed. This fixed-angle model arises in chemistry [CH88] and in particular in protein folding (see Section 9.7). Alternatively, a fixed angle can be simulated by adding bars between vertices of distance two along the polygon. The configuration space behaves similarly to the planar case:

THEOREM 9.2.4 Fixed-angle equilateral 3D polygons [CJ]

Let $\mathcal{M}$ be the configuration space of an equilateral polygon with $n \geq 6$ equal bar lengths and fixed equal angles, modulo congruences of $\mathbb{R}^{3}$. Suppose further that every turn angle is within an additive $\epsilon$ of $2 \pi / n$ for $\epsilon$ sufficiently small (i.e., configurations are forced nearly planar). Then $\mathcal{M}$ has at most two components. When $n$ is odd, $\mathcal{M}$ is a smooth manifold of dimension $n-6$. When $n$ is even, $\mathcal{M}$ is singular.

When $n=6$, the underlying graph is the graph of an octahedron, and there are cases when it is rigid and cases when it is not. This linkage corresponds to cyclohexane in chemistry, and its flexibility was studied by [Bri96] and [Con78].

The restriction of the polygon configurations being almost planar leads to the following problem:

PROBLEM 9.2.5 General equilateral equi-angular 3D polygons [Cri92]

How many components does $\mathcal{M}$ have in the theorem above if $\epsilon$ is allowed to be large?

\subsection{CONFIGURATION SPACES WITHOUT SELF-INTER- SECTIONS}

When the linkage is not permitted to self-intersect, the main question that has been studied is when it can be locked. Three main classes of linkages have been studied in this context: arcs, cycles, and trees. When the linkage is planar and has cycles, 
we assume that the clockwise/counterclockwise orientation is given and fixed, for otherwise the linkage is trivially locked: no cycle can be "flipped over" in the plane without self-intersection.

\section{GLOSSARY}

Locked linkage: A linkage whose configuration space has multiple connected components when self-intersections are disallowed.

Lockable class of linkages: There is a locked linkage in the class.

Unlockable class of linkages: No linkage in the class is locked.

FIGURE 9.3.1

The problems of arc straightening, cycle convexifying, and tree flattening.

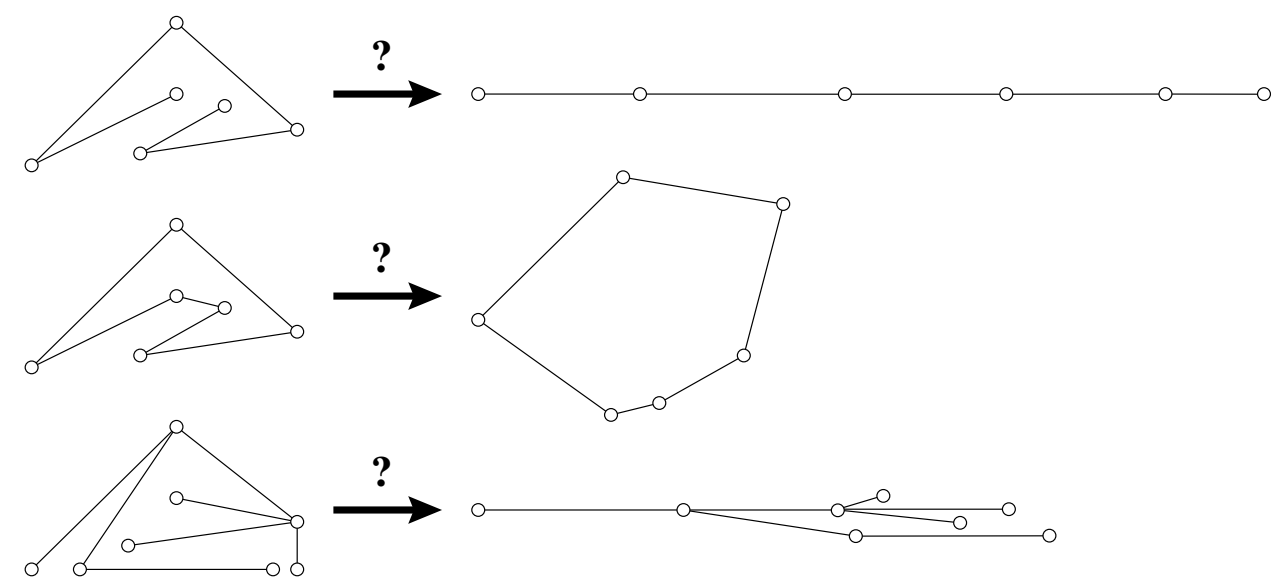

Straightening an arc: A motion bringing a polygonal arc from a given configuration to its straight configuration in which every joint angle is $\pi$.

Convexifying a cycle: A motion bringing a polygonal arc from a given configuration to a convex configuration in which every joint angle is at most $\pi$.

Flattening a tree: A motion bringing a polygonal tree from a given configuration to a flat configuration in which every joint angle is either $0, \pi$, or $2 \pi$, and every bar points "away" from a designated root node.

\section{WHICH LINKAGES ARE LOCKED?}

Which of the main classes of linkages can be locked is summarized in Table 9.3.1. In short, the existence of locked arcs and locked unknotted cycles is equivalent to the existence of knots in that dimension: just in 3D. However, this equivalence is 
by no means obvious, especially in $2 \mathrm{D}$, as evidenced by the existence of knotted trees in $2 \mathrm{D}$.

TABLE 9.3.1 Summary of what types of linkages can be locked.

\begin{tabular}{|l|c|c|}
\cline { 2 - 3 } \multicolumn{1}{c|}{} & ARCS AND CYCLES & TREES \\
\hline 2D & Not lockable $[$ CDR, Str00, CDIO02] & Lockable $\left[\right.$ BDD $^{+} 02$, CDR02] \\
\hline 3D & Lockable $\left[\right.$ CJ98, BDD ${ }^{+} 01$, Tou01] & Lockable $[$ arcs are a special case] \\
\hline 4D ${ }^{+}$ & Not lockable $[$CO01] & Not lockable [CO01] \\
\hline
\end{tabular}

One main approach for determining whether a linkage is locked is to consider the equivalent problem of finding a motion from any configuration to a canonical configuration. Because linkage motions are reversible and concatenable, if every configuration can be canonicalized, then every configuration can be brought to any other configuration, routing through the canonical configuration. Conversely, if some configuration cannot be canonicalized, then we know a pair of configurations that cannot reach each other, and therefore the linkage is locked.

This idea leads to the notions of straightening arcs, convexifying cycles, and flattening trees, as defined above. There is only one straight configuration of an arc, but there are multiple convex configurations of cycles and flat configurations of trees; fortunately, it is fairly easy to reconfigure between any pair of convex configurations of a cycle $\left[\mathrm{ADE}^{+} 01\right]$ or between any pair of flat configurations of a tree $\left[\mathrm{BDD}^{+} 02\right]$.

\section{LOCKED LINKAGES}

The first results along these lines were negative (see Figure 9.3.2): polygonal arcs in 3D and unknotted polygonal cycles in 3D can be locked [CJ98], and planar polygonal trees can be locked $\left[\mathrm{BDD}^{+} 02\right]$. Since these results, other examples of unknotted but locked $3 \mathrm{D}$ polygonal cycles $\left[\mathrm{BDD}^{+} 01\right.$, Tou01] and locked 2D polygonal trees [CDR02] have been discovered.

More generally and recently, Alt, Knauer, Rote, and Whitesides [AKRW03] constructed a large family of locked $2 \mathrm{D}$ trees and $3 \mathrm{D}$ arcs in which it is PSPACEhard to determine whether one configuration can reach another configuration via a continuous motion that avoids self-intersection. Their construction combines several gadgets, many of which resemble the examples in Figure 9.3.2, as well as the "interlocked" linkages of [DLOS, DLOS02]. However, this work leaves open a closely related problem, deciding whether every pair of configurations can reach each other:

PROBLEM 9.3.1 Complexity of testing whether a linkage is locked $\left[\mathrm{BDD}^{+} 01\right]$ What is the complexity of deciding whether a linkage is locked? Particular cases of interest are $3 D$ arcs, unknotted $3 D$ cycles, and $2 D$ trees. 
FIGURE 9.3.2

Known examples of locked linkages.

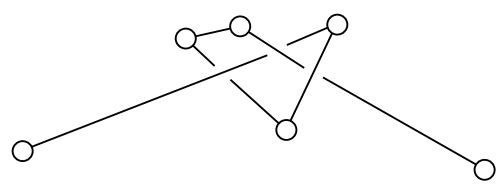

3D arc [CJ98]

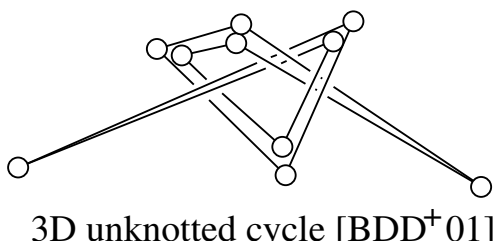

$3 \mathrm{D}$ unknotted cycle $\left[\mathrm{BDD}^{+} 01\right]$

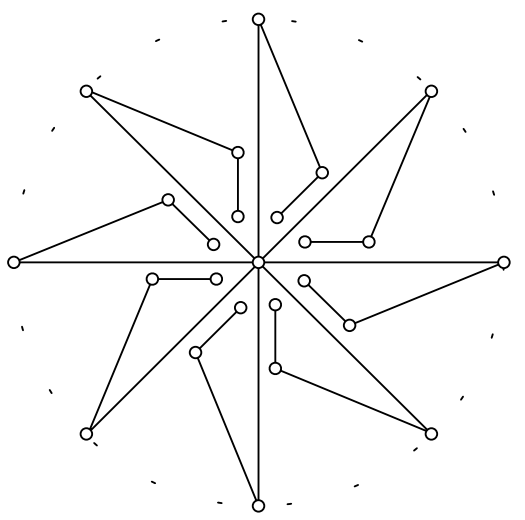

$2 \mathrm{D}$ tree $\left[\mathrm{BDD}^{+} 021\right.$

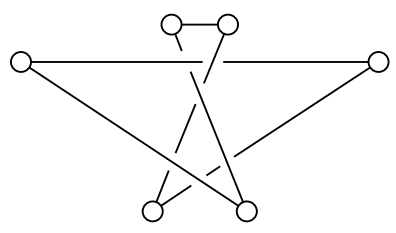

3D unknotted cycle [CJ98]

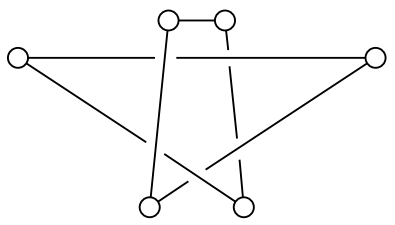

3D unknotted cycle [Tou01]

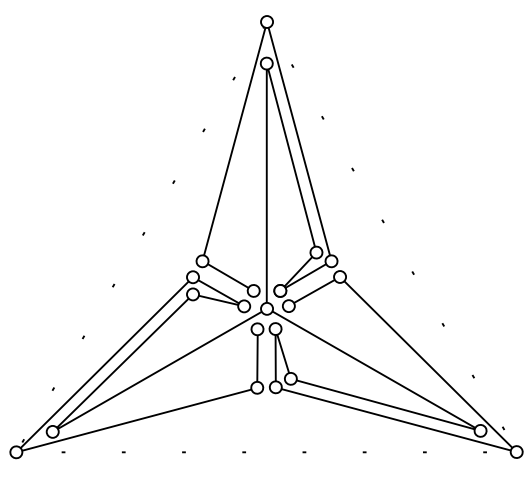

$2 \mathrm{D}$ tree $[\mathrm{CDR} 021$

\section{UNLOCKED LINKAGES}

Unlockability was first established in 4D and higher [CO01], where one-dimensional arcs, cycles, and trees have so much freedom that they can never lock. Intuitively, the barriers (self-intersecting configurations) that might prevent e.g. straightening the vertex between the first two bars of an arc have dimension at least 2 lower than the configuration space of that vertex, and hence all barriers can be avoided. Thus, the only problem with straightening an arc vertex-by-vertex is that the configuration that results from straightening one extreme vertex might have self-intersections; in this case, the linkage can be perturbed to remove the problem. Convexifying cy- 
cles in 4D and higher is more difficult, but follows a similar idea.

The last cell of Table 9.3.1 to be filled was that $2 \mathrm{D}$ arcs and cycles never to lock [CDR]. Indeed, the following more general theorem holds:

THEOREM 9.3.2 Straightening $2 D$ arcs and convexifying $2 D$ cycles [CDR] Given a disjoint collection of polygonal arcs and polygonal cycles in the plane, there is a motion that avoids self-intersection and, after finite time, straightens every outermost arc and convexifies every outermost cycle. (An arc or cycle is outermost if it is not contained within another cycle.)

In this theorem, arcs and cycles contained within other cycles may not straighten or convexify - they simply "come along for the ride" — but this is the best we could hope for in general.

There are now three methods for solving this problem. See Figure 9.3.3 for a visual comparison on a simple example. The first method is based on flow through an ordinary differential equation defined implicitly by a convex optimization problem [CDR]. The second method is more combinatorial and is based on algebraic motions defined by single-degree-of-freedom mechanisms given by pseudotriangulations [Str00]. The third method is based on energy minimization via gradient descent [CDIO02].

The first two motions have the additional property of being expansive - the distance between every pair of vertices never decreases over time - while the third motion only relies on the existence of such a motion. The first and last motions, being flow-based, preserve any initial symmetries of the linkage. Characterizing by continuity, the three motions are respectively piecewise- $C^{1}$, piecewise- $C^{\infty}$, and $C^{\infty}$. Only the last motion has a corresponding finite-time algorithm to compute a motion that is piecewise-linear through configuration space, i.e., the motion can be decomposed into steps where each angle in each step changes at a constant rate. This algorithm is also easy to implement.

\section{SPECIAL CLASSES OF LINKAGES}

In addition to these results for general classes of linkages, various special classes have been shown to have different properties. Polygonal arcs in 3D that lie on the surface of a convex polyhedron, or having a non-self-intersecting orthogonal projection, are never locked $\left[\mathrm{BDD}^{+} 01\right]$. Polygonal cycles in $3 \mathrm{D}$ having a non-selfintersecting orthogonal projection are also never locked $\left[\mathrm{CKM}^{+} 01\right]$.

\section{FLIPS AND FLIPTURNS}

One of the first papers essentially about unlocking linkages is by Erdős [Erd35], who asked whether a particular "flipping" algorithm always convexifies a planar polygon by motions through $3 \mathrm{D}$ in a finite number of steps. A flip rotates by $180^{\circ}$ a subchain of the polygon, called a pocket, whose endpoints are consecutive vertices along the convex hull of the polygon. Each such flip never causes the 
FIGURE 9.3.3

Convexifying a common polygon via all three convexification methods.

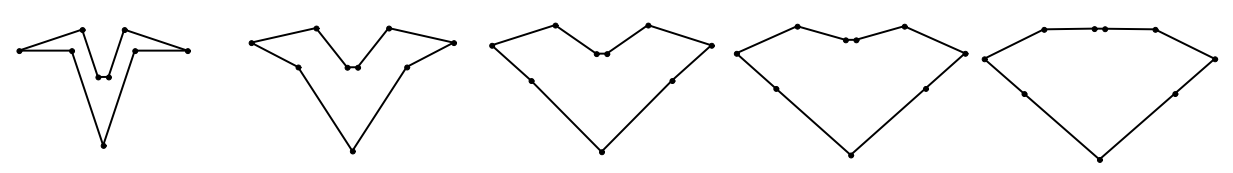

(a) Via convex programming $[\mathrm{CDR}]$
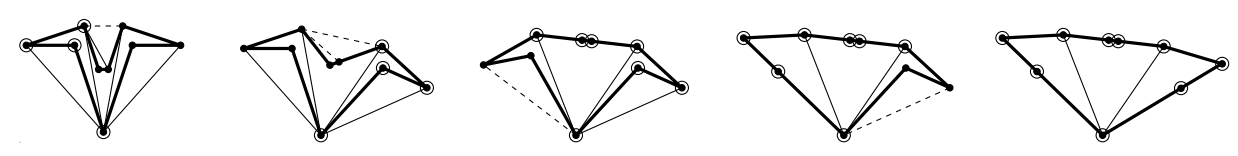

(b) Via pseudotriangulations [Str00]. Pinned vertices are circled.
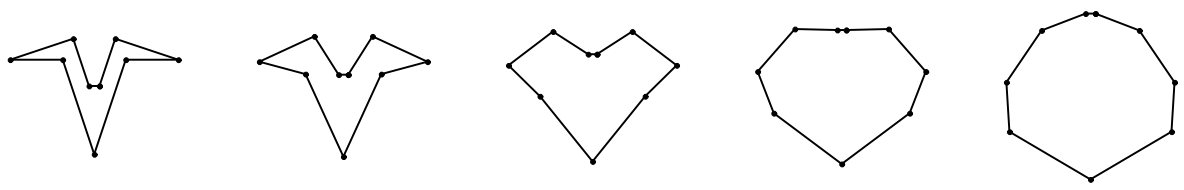

(c) Via energy minimization [CDIO02].

polygon to self-intersect. ${ }^{1}$ Nagy [Nag39] was the first to prove that a polygon admits only finitely many flips before convexifying. Thus, pocket flipping is one suitable strategy for convexifying a $2 \mathrm{D}$ polygon by motions in $3 \mathrm{D}$. This result was subsequently rediscovered several times; see [Tou99, Grü95].

Joss and Shannon (1973) first proved that the number of flips required to convexify a polygon cannot be bounded in terms of the number of vertices, but this work remains unpublished; see [Grü95, Tou99]. However, it may still be possible to bound the number of flips using other metrics:

PROBLEM 9.3.3 Bounding the number of flips [M. Overmars, Feb. 1998]

Bound the maximum number of flips a polygon admits in terms of natural measures of geometric closeness such as the sharpest angle, the diameter, and the minimum distance between two nonincident edges.

A related computational problem is to compute the extreme numbers of flips:

\footnotetext{
${ }^{1}$ Erdős [Erd35] originally proposed flipping multiple pockets at once, but such an operation can lead to self-intersection; Nagy [Nag39] fixed this problem by proposing flipping only one pocket at once.
} 
FIGURE 9.3.4

Flipping a polygon until it is convex.
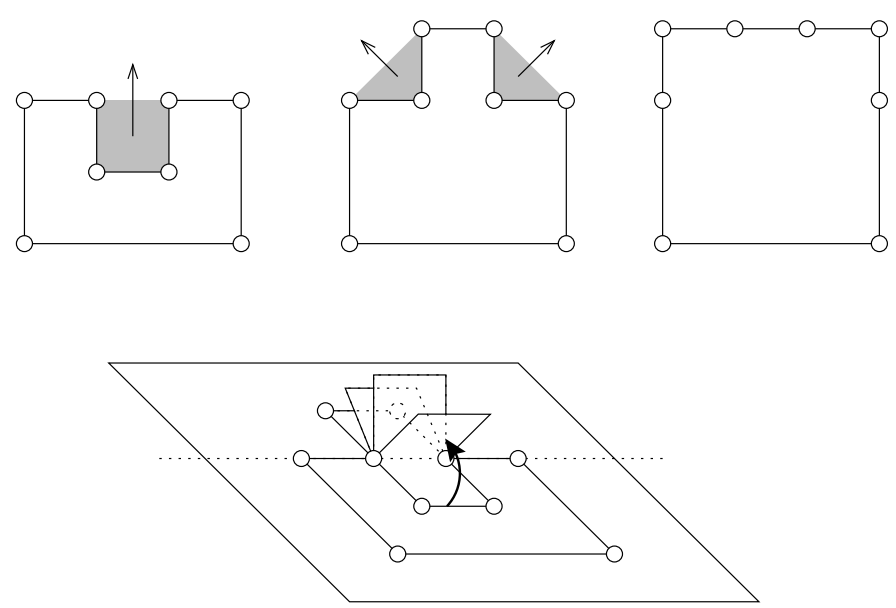

PROBLEM 9.3.4 Maximizing or minimizing flips [Dem02]

What is the complexity of minimizing or maximizing the length of a convexifying sequence of flips for a given polygon?

Several variations on flips have also been considered. Grünbaum and Zaks [GZ01] generalized Nagy's results to polygons with self-intersections; still they can be convexified by finitely many flips. Wegner [Weg93] introduced the notion of deflations, which are the exact reverse of flips, and Fevens et al. $\left[\mathrm{FHM}^{+} 01\right]$ showed that some polygons admit infinitely many deflations.

Flipturns are similar to flips, except that the pocket is temporarily severed from the rest of the linkage and rotated $180^{\circ}$ in the plane around the midpoint of the hull edge. Such an operation is not a valid linkage motion, but it has the advantage that the number of flipturns that a polygon admits before convexification is $O\left(n^{2}\right)$ $\left[\mathrm{ACD}^{+} 00, \mathrm{ABC}^{+} 00\right]$. This bound is tight up to a constant factor [Bie00], and there is extensive work on finding the precise constants $\left[\mathrm{ACD}^{+} 00\right]$, though some gaps remain to be closed. Also, related to Problem 9.3.4, it is known that maximizing the length of a convexifying flipturn sequence is weakly NP-hard $\left[\mathrm{ACD}^{+} 00\right]$. Minimizing the number of flipturns leads to the following interest problem:

PROBLEM 9.3.5 Number of required flipturns [Bie00]

Is there a polygon that requires $\Omega\left(n^{2}\right)$ flipturns to convexify, or can all polygons be convexified by $\left(n^{2}\right)$ carefully chosen flipturns?

The best known lower bound is $\Omega(n)$.

\section{INTERLOCKED LINKAGES}

Combinations of polygonal arcs and cycles in 3D that can or cannot be locked (or 
more accurately, "interlocked") are studied in [DLOS, DLOS02]. More precisely, this work studies the shortest (fewest-bar) 3D arcs and cycles that can interlock with each other. For example, three 3 -arcs (arcs with three bars each) can interlock, as can a 3-arc and a 4-arc, or a 3-cycle and a 4-arc, or a 3-arc and a 4-cycle. However, two 3-arcs and arbitrarily many 2-arcs never interlock, and nor can a 3-cycle and a 3-arc. Also considered in [DLOS02] is the case that some of the pieces have restricted motion, e.g., all angles being fixed, or just rigid motions being allowed.

\subsection{UNIVERSALITY RESULTS}

\section{TRACING CURVES}

The classic motivation of building linkages is to design a planar linkage in which one of the vertices traces a portion of a desired curve given by some polynomial function. In particular, Watt posed the problem of finding a linkage with some vertices pinned so that one vertex would trace out a line (segment). Watt's problem, at first thought to be impossible, was finally solved by Peaucellier in [Pea73], as well as by Lipkin in [Lip71]. See also [Kem77] and [Har74].

Later, Kempe [Kem76] described a linkage that would trace out a portion of any algebraic curve in the plane. However, his description is very brief and it leaves unspecified what portion of the algebraic curve is actually traced out, and whether there are other, possibly unwanted components or pieces of other algebraic curves that can also be traced out. This question also arises for the linkages that trace a line segment.

\section{GLOSSARY}

Real algebraic set: A subset of $\mathbb{R}^{N}$ given by a finite number of polynomial equations with real coefficients.

Real semi-algebraic set: A subset of $\mathbb{R}^{N}$ given by a finite number of polynomial equations and inequalities with real coefficients.

It is important to realize the distinction between an algebraic set and a semialgebraic set. For example, a circle (excluding its interior) is an algebraic set, while a (closed) line segment is a semi-algebraic set but not an algebraic set. The linear projection of an algebraic set is always a semi-algebraic set, but it may not be an algebraic set. The configuration space of a linkage is an algebraic set, but the locus of possible positions of one of its vertices is only guaranteed to be a semi-algebraic set, because it represents the projection onto the coordinates corresponding to one of the vertices of the linkage.

\section{ARBITRARY CONFIGURATION SPACES}


One of the more precise results related to Kempe's result is the following:

THEOREM 9.4.1 Creating linkage configuration spaces [KM95]

Let $M$ be any compact smooth manifold. Then there is a planar linkage whose configuration space is diffeomorphic to a disjoint union of some number of copies of $M$.

This result was also claimed by Thurston, but there does not seem to be a written proof by him. As a consequence of this result, we obtain the following precise version of what Kempe was trying to claim. This consequence is proved by King [Kin99] using the techniques of Kapovich-Millson [KM02] and Thurston.

THEOREM 9.4.2 Tracing out an algebraic curve [Kin99]

Let $X$ be any set in the plane that is the polynomial image of a closed interval. Then there is a linkage in the plane with some pinned vertices such that one of the vertices traces out $X$ exactly.

See [JS99, BM56] for other discussions of how to create linkages to trace out at least a portion of a given algebraic curve. King [Kin] also generalizes this result to higher dimensions and to the semialgebraic sets arising from projecting the configuration space down to consider some subset of the vertices. See also [KM02] for connections to universality theorems concerning configuration spaces of lines in the plane, for example, as in the work of [Mnë88]. Finally, the complexity results of [HJW85] described in Section 9.5 build off a universality construction similar to those mentioned above.

\subsection{COMPUTATIONAL COMPLEXITY}

There are a variety of algorithmic questions that can be asked about a given linkage. Most of these questions are computationally difficult to answer, either NP-hard or PSPACE-hard. Nonetheless, given the importance of these problems, there is work on developing (exponential-time) algorithms.

\section{GLOSSARY}

Ruler folding problem: Given a polygonal arc (i.e., a sequence of bar lengths) and a desired length $L$, is there a configuration of the arc (ruler) in which the bars lie along a common line segment of length $L$ ? If so, find such a configuration. (The problem can also be phrased as reconfiguration, provided the linkage is permitted to self-intersect.)

Reachability problem: Given a configuration of a linkage, a distinguished vertex, and a point in the plane, is it possible to reconfigure the linkage so that the distinguished vertex touches the given point? If so, find such a reconfiguration. In this problem, the linkage has one or more vertices pinned to particular locations in the plane. 
Reconfiguration problem: Given two configurations of a linkage, is it possible to reconfigure one into the other? If so, find such a reconfiguration.

Locked decision problem: Given a linkage, is it locked?

\section{HARDNESS RESULTS}

One of the simplest complexity results is about the ruler folding problem, obtained via a reduction from set partition:

THEOREM 9.5.1 Complexity of ruler folding [HJW85]

The ruler folding problem is NP-complete.

Building on this result, the same authors establish

\section{THEOREM 9.5.2 Complexity of arc reachability [HJW85]}

The reachability problem is NP-hard for a planar polygonal arc in the presence of four line-segment obstacles and permitting the arc to self-intersect.

For general linkages instead of arcs, stronger complexity results exist:

THEOREM 9.5.3 Complexity of reachability [HJW84]

The reachability problem is PSPACE-hard for a planar linkage without obstacles and permitting the linkage to self-intersect.

On the other hand, a similar result holds for a polygonal arc among obstacles:

THEOREM 9.5.4 Complexity of arc reachability among obstacles [JP85]

The reachability problem is PSPACE-hard for a planar polygonal arc in the presence of polygonal obstacles and permitting the arc to self-intersect.

Finally, when the linkage is not permitted to self-intersect, and there are no obstacles, hardness is known in cases when the linkage can be locked; see Section 9.3.

THEOREM 9.5.5 Complexity of non-self-intersecting arc reconfiguration [AKRW03]

The reconfiguration problem is PSPACE-hard for a 3D polygonal arc or a $2 D$ polygonal tree when the linkage is not permitted to self-intersect.

\section{ALGORITHMS}

Algorithms for linkage reconfiguration problems can be obtained from general motionplanning results in Chapter 47 (Section 47.1.1). This connection seems to have only recently been made explicit [AKRW03]. To apply the roadmap algorithm of Canny [Can87] (Theorem 47.1.2), we first phrase the algorithmic linkage problems into the motion-planning framework. 
The configuration space of a given linkage is the subset of $\mathbb{R}^{v c}$ in which every point satisfies certain bar-length constraints and, if desired, non-intersection constraints between all pairs of bars. Both types of constraints can be phrased using constant-degree polynomial equations and inequalities, e.g., the former by setting the squared length of each bar to the desired value. (There are also embeddings of the configuration space into Euclidean space with fewer than $v c$ dimensions, dependent on the number of degrees of freedom in the linkage, but the $v c$-dimensional parameterization is most naturally semi-algebraic.)

Returning to the motion-planning framework, the polynomial equations and inequalities are precisely the obstacle surfaces. The configuration space has dimension $k=v c$, and there are $n \leq b^{2}$ obstacle surfaces where $b$ is the number of bars, each with degree $d=O(1)$. We can factor out the trivial rigid motions by supposing that one bar of the linkage is pinned, reducing $k$ to $(v-2) c$. Now running the roadmap algorithm produces a representation of the entire configuration space. By path planning within this space, we can solve the reconfiguration problem. By a simple pass through the representation, we can tell whether the space is connected, solving the locked decision problem. By slicing the space with a polynomial specifying that a particular vertex is at a particular point in the plane, we can solve the reachability problem.

Plugging $k \leq v c, n \leq b^{2}$, and $d=O(1)$ into the roadmap algorithm with deterministic running time $O\left(n^{k}(\log n) d^{O\left(k^{4}\right)}\right)$ and randomized expected running time $O\left(n^{k}(\log n) d^{O\left(k^{2}\right)}\right)$, we obtain

\section{COROLLARY 9.5.6 Roadmap algorithm applied to linkages [AKRW03]}

The reachability, reconfiguration, and locked decision problems can be solved for an arbitrary linkage with $v$ vertices and $b$ bars in $\mathbb{R}^{c}$ using $O\left(b^{2 v c}(\log b) 2^{O(v c)^{4}}\right)$ deterministic time or $O\left(b^{2 v c}(\log b) 2^{O(v c)^{2}}\right)$ expected randomized time.

\subsection{KINEMATICS}

According to Bottema and Roth [BR79b], "kinematics is that branch of mechanics which treats the phenomenon of motion without regard to the cause of the motion. In kinematics there is no reference to mass or force; the concern is only with relative positions and their changes." Kinematics is a subject with a long history and which has had, at various times, notable influence on and has to some extent has been partially identified with such areas as algebraic geometry, differential geometry, mechanics, singularity theory, and Lie theory. It has often been a subject studied from an engineering point of view, and there are many detailed calculations with respect to particular mechanisms of interest. As a representative example, we consider four-bar mechanisms (Figure 9.6.1):

\section{GLOSSARY}

Mechanism: A linkage with one degree of freedom, modulo global translation 
FIGURE 9.6.1

The coupler curve of the midpoint $E$ of the coupler $C D$ as it moves relative to the frame $A B$ in a four-bar mechanism.

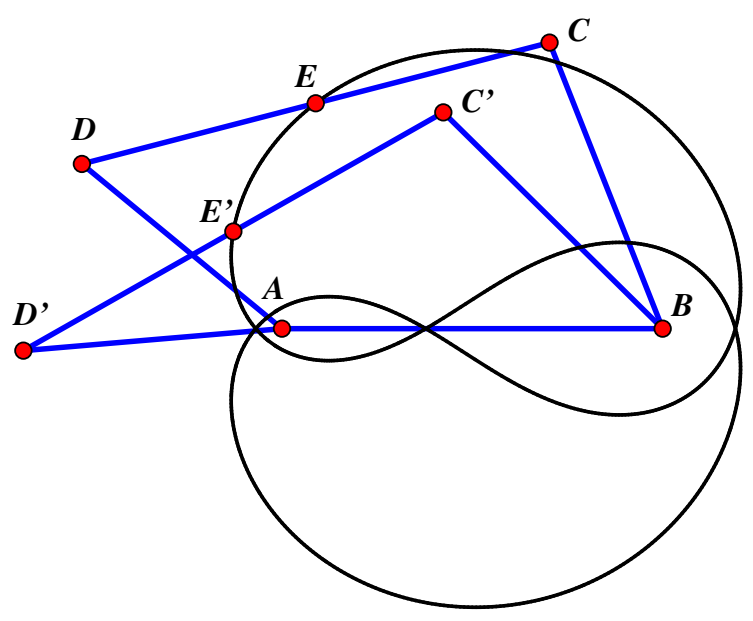

and rotation.

Four-bar mechanism: A four-bar polygonal cycle; see Figure 9.6.1 for an example. Sometimes called a three-bar mechanism.

Frame: We generally fix a frame of reference for a mechanism by pinning one bar, fixing its position in the plane. This bar is called the frame. In Figure 9.6.1, bar $A B$ is pinned.

Coupler: A distinguished bar other than the frame. In Figure 9.6.1, we consider the coupler $C D$.

Coupler motion: The motion of the entire plane induced by the relative motion of the coupler with respect to the frame.

Coupler curve: The path traced during the coupler motion by any point rigidly attached to the coupler (e.g., via two additional bars). Figure 9.6.1 shows the coupler curve of the midpoint $E$ of the coupler bar $C D$.

\section{FOUR-BAR MECHANISM}

Coupler curves can be surprisingly complex. In the generic case, a coupler curve of a four-bar mechanism is an algebraic curve of degree 6. Substantial effort has been put into cataloging the different shapes of coupler curves that can arise from four-bar and other mechanisms. A sample theorem in this context is the following:

THEOREM 9.6.1 Multiplicity of coupler curves [Rob75]

Any coupler curve of a four-bar mechanism can be generated by two other four-bar mechanisms.

\section{GLOSSARY}

Infinitesimal motion or first-order flex: The first derivative of a motion at an 
instantaneous moment in time, assigning a velocity vector to each point involved in the motion. (See Chapter 49 for a more thorough explanation in the context of rigidity.)

Pole or instantaneous pole: The instantaneous fixed point of a first-order motion of the plane. For a rotation, the pole is the center of rotation. For a translation, the pole is a point at infinity in the projective plane. A combination of rotation and translation can be rewritten as a pure rotation.

Polode: The locus of poles over time during a motion of the plane.

\section{POLES}

Some of the central theorems in kinematics treat the instantaneous case. Poles characterize the first-order action of a motion at each moment in time. Together, the polode can be viewed relative to either the fixed plane of the frame (the fixed polode) or the moving plane of the coupler (moving polode). Apart from degenerate cases, a planar motion can be described by the moving polode rolling along the fixed polode. A basic theorem in the context of poles is the following:

THEOREM 9.6.2 Three-Pole Theorem

For any three motions of the plane, the instantaneous poles of the three mutual relative motions are collinear at any moment in time.

\section{FURTHER READING}

For a general introduction and sampling of the field of kinematics, see [Hun78, BR79b, Sta97, McC90, Pot94, McC00]. For relations to singularity theory, see e.g. [GHM97]. For examples, analysis, and synthesis of specific mechanisms such as the four-bar mechanism, see [GN86, Mik01, Sta99, Ale95, BS90, Leb67, Con79, Con78]. For some typical examples from an engineering viewpoint, see e.g. [CP91, Che02, Ler00]. See also Section 59.4 of this Handbook.

\subsection{APPLICATIONS}

Applications of linkages arise throughout science and engineering. We highlight three modern applications: robotics, manufacturing, and protein folding.

\section{APPLICATIONS IN ENGINEERING}

The study of linkages in fact originated in the context of mechanical engineering, e.g., for the purpose of converting circular motion into linear motion. Today, one of the driving applications for linkages is robotics, in particular robotic arms.

A robotic arm can be modeled as a linkage, typically a polygonal chain. Some 
robotic arms have hinges that force the bars to remain coplanar, modeled by $2 \mathrm{D}$ chains; other arms have universal joints, modeled by 3D chains; other arms pose additional different constraints (such as incident bars being coplanar, yet the whole linkage need not be coplanar), leading to other models of linkage folding. Some planar robotic arms reserve slightly offset planar planes for the bars, modeled by a planar polygonal chain that permits self-intersection. Most other robotic arms are modeled by disallowing self-intersection.

The reachability problem is largely motivated by robotic arms, where the "hand" at one end of the arm must be placed at a particular location, e.g., to pick up an object, but the rest of the configuration is secondary. In other contexts, the entire configuration of the arm is important, and we need to plan a motion to a target configuration, leading to the reconfiguration problem. The locked decision problem is the first question one might ask about the simplicity/complexity of motion planning for a particular type of linkage. However, all of these problems are typically studied in the context of linkages without obstacles, but in robotics there are almost always obstacles. Some obstacles, such as a halfplane representing the floor, can often be avoided; but more generally the problems become much more complicated. See Chapter 47.

Another area with linkage applications is manufacturing. Given a straight hydraulic tube or piece of wire, a typical goal is to produce a desired folded configuration. In these contexts, we want to bend the wire as little as possible. In particular, a typical constraint is to bend the wire only monotonically: once it is bent one way, it cannot be bent the other way. This constraint forces straight segments of the target shape to remain straight throughout the motion. Thus, the problem can be modeled as straightening a polygonal chain, either in 2D or $3 \mathrm{D}$ depending on the application, with additional constraints. For example, the expansive motions described in Section 9.3 fold all joints monotonically; however, their reliance on bending most joints simultaneously may be undesirable. Arkin et al. [AFMS01] consider the restriction in which only a single joint can be rotated at once, together with additional realistic constraints arising in wire bending.

\section{APPLICATIONS IN BIOLOGY}

A crude model of a protein backbone is a polygonal chain in $3 \mathrm{D}$, and a similarly crude model of an entire protein is a polygonal tree in 3D. In both cases, the vertices represent atoms, and the bars represent bonds between atoms (which in reality stay roughly the same length). In proteins, these bar/bond lengths are typically all within a factor of 2 of each other. Two atoms cannot occupy the same space, which can be roughly modeled by disallowing self-intersection. One interesting open problem in this context is the following:

PROBLEM 9.7.1 Equilateral or near-equilateral locked linkages $\left[\mathrm{BDD}^{+} 01\right]$ Is there a locked equilateral arc, cycle, or tree in 3D? More generally, what is the smallest value of $\alpha \geq 1$ for which there is a locked arc/cycle/tree in $3 D$ with all edge lengths between 1 and $\alpha$ ?

These crude models may lead to some biological insight, but they do not capture 
several aspects of real protein folding.

One aspect that can easily be incorporated into linkage folding is that the angles between incident bars is typically fixed. This fixed-angle constraint can alternatively be viewed as adding bars between vertices originally at distance two from each another. Soss et al. [Sos01, SEO01, ST00] initiated study of such fixedangle linkages in computational geometry, in particular establishing NP-hardness of deciding reconfigurability or flattenability. Aloupis et al. $\left[\mathrm{ADD}^{+} 02, \mathrm{ADM}^{+} 02\right]$ consider when fixed-angle linkages are not locked in the sense that all flat states are reachable from each other by motions avoiding self-intersection.

A more challenging aspect of protein folding is the thermodynamic hypothesis [Anf72]: that folding is encouraged to follow energy-minimizing pathways. Indeed, the bars are not strictly binding, nor are they completely fixed in length; they are merely encouraged to do so, and sometimes violate these constraints. Unfortunately, these properties are different to model, and energy functions defined so far are either incomplete or difficult to manipulate. Also, the implications on linkage-folding problems remain unclear.

One particularly simple energy-based model of protein folding that has received substantial attention in computer science and biology is the HP (HydrophilicHydrophobic) model; see e.g. [ABD ${ }^{+}, \mathrm{CD} 93$, Dil90, Hay98]. This model is particularly discrete, modeling a protein as an equilateral chain on a lattice, typically square or cubic grid, but possibly also a triangular or tetrahedral lattice. The model captures only hydrophobic bonds and forces, clustering to avoid external water. Finding the optimal folding even in this simple model is NP-complete $\left[\mathrm{BL98}, \mathrm{CGP}^{+} 98\right]$, though there are several constant-factor approximation algorithms [HI96, New02, $\mathrm{ABD}^{+}$97]. One interesting open problem is whether designing a protein to fold into particular shape is easier than finding the shape to which a particular protein folds $\left[\mathrm{ABD}^{+}\right]$:

PROBLEM 9.7.2 HP protein design $\left[\mathrm{ABD}^{+}\right]$

What is the complexity of deciding whether a given subset of the lattice is an optimal folding of some HP protein, and if so finding such a protein? What if it must be the unique optimal folding of the HP protein?

A result related to the second half of this problem is that arbitrarily long HP proteins with unique optimal foldings exist, at least for open and closed chains in a $2 \mathrm{D}$ square grid $\left[\mathrm{ABD}^{+}\right]$.

\subsection{SOURCES AND RELATED MATERIAL}

\section{FURTHER READING}

[O'R98, Dem00, Dem02]: Surveys on folding and unfolding problems in general, which includes linkage folding in particular. 


\section{RELATED CHAPTERS}

Chapter 32: Computational topology

Chapter 33: Computational real algebraic geometry

Chapter 47: Algorithmic motion planning

Chapter 48: Robotics

Chapter 49: Computer graphics

Chapter 55: Manufacturing processes

Chapter 59: Geometric applications of the Grassmann-Cayley algebra

Chapter 60: Rigidity and scene analysis

Chapter 63: Biological applications of computational topology

\section{REFERENCES}

$\left[\mathrm{ABC}^{+} 00\right]$ Hee-Kap Ahn, Prosenjit Bose, Jurek Czyzowicz, Nicolas Hanusse, Evangelos Kranakis, and Pat Morin. Flipping your lid. Geombinatorics, 10(2):57-63, 2000.

$\left[\mathrm{ABD}^{+}\right] \quad$ Oswin Aichholzer, David Bremner, Erik D. Demaine, Henk Meijer, Vera Sacristán, and Michael Soss. Long proteins with unique optimal foldings in the H-P model. Comput. Geom. Theory Appl. To appear.

$\left[\mathrm{ABD}^{+}\right.$97] Richa Agarwala, Serafim Batzoglou, Vlado Dancik, Scott E. Decatur, Martin Farach, Sridhar Hannenhalli, S. Muthukrishnan, and Steven Skiena. Local rules for protein folding on a triangular lattice and generalized hydrophobicity in the HP model. Journal of Computational Biology, 4(2):275-296, 1997.

$\left[\mathrm{ACD}^{+} 00\right]$ Oswin Aichholzer, Carmen Cortés, Erik D. Demaine, Vida Dujmović, Jeff Erickson, Henk Meijer, Mark Overmars, Belén Palop, Suneeta Ramaswami, and Godfried T. Toussaint. Flipturning polygons. In Proc. Japan Conf. Discrete Comput. Geom., Lecture Notes in Comput. Sci., Tokyo, Japan, Nov. 2000. To appear in Discrete $E^{6}$ Computational Geometry.

$\left[\mathrm{ADD}^{+} 02\right]$ Greg Aloupis, Erik D. Demaine, Vida Dujmović, Jeff Erickson, Stefan Langerman, Henk Meijer, Ileana Streinu, Joseph O'Rourke, Mark Overmars, Michael Soss, and Godfried T. Toussaint. Flat-state connectivity of linkages under dihedral motions. In Proc. Internat. Symp. Algorithms and Computation, Lecture Notes in Comput. Sci., pages 369-380, Vancouver, Canada, Nov. 2002.

$\left[\mathrm{ADE}^{+} 01\right]$ Oswin Aichholzer, Erik D. Demaine, Jeff Erickson, Ferran Hurtado, Mark Overmars, Michael A. Soss, and Godfried T. Toussaint. Reconfiguring convex polygons. Comput. Geom. Theory Appl., 20(1-2):85-95, Oct. 2001.

$\left[\mathrm{ADM}^{+}\right.$02] Greg Aloupis, Erik D. Demaine, Henk Meijer, Joseph O'Rourke, Ileana Streinu, and Godfried Toussaint. Flat-state connectedness of fixed-angle chains: Special acute chains. In Proc. 14th Canadian Conf. Comput. Geom., pages 27-30, Lethbridge, Canada, Aug. 2002.

[AFMS01] Esther M. Arkin, Sándor P. Fekete, Joseph S. B. Mitchell, and Steven S. Skiena. On the manufacturability of paperclips and sheet metal structures. In Proceedings 
of the 17th European Workshop on Computational Geometry, pages 187-190, Berlin, Germany, Mar. 2001.

[AKRW03] Helmut Alt, Christian Knauer, Guenter Rote, and Sue Whitesides. The complexity of (un)folding. In Proc. 19th Sympos. Comput. Geom., San Diego, California, June 2003. To appear.

[Ale95] V. A. Aleksandrov. A new example of a bendable polyhedron. Sibirsk. Mat. Zh., 36(6):1215-1224, i, 1995.

[Anf72] Christian Anfinsen. Studies on the principles that govern the folding of protein chains. In Les Prix Nobel en 1972, pages 103-119. Nobel Foundation, 1972.

$\left[\mathrm{BDD}^{+} 01\right]$ T. Biedl, E. Demaine, M. Demaine, S. Lazard, A. Lubiw, J. O'Rourke, M. Overmars, S. Robbins, I. Streinu, G. Toussaint, and S. Whitesides. Locked and unlocked polygonal chains in three dimensions. Discrete Comput. Geom., 26(3):269281, Oct. 2001. The full version is Technical Report 060, Smith College, 1999, and arXiv:cs.CG/9910009, http://www.arXiv.org/abs/cs.CG/9910009.

$\left[\mathrm{BDD}^{+} 02\right]$ Therese Biedl, Erik Demaine, Martin Demaine, Sylvain Lazard, Anna Lubiw, Joseph O'Rourke, Steve Robbins, Ileana Streinu, Godfried Toussaint, and Sue Whitesides. A note on reconfiguring tree linkages: Trees can lock. Discrete Appl. Math., 117(13):293-297, 2002. The full version is Technical Report SOCS-00.7, School of Computer Science, McGill University, September 2000, and Computing Research Repository paper cs.CG/9910024. http://www.arXiv.org/abs/cs.CG/9910024.

[Bie00] Therese Biedl. Polygons needing many flipturns. Technical Report CS-2000-04, Dept. of Computer Science, U. Waterloo, 2000. ftp://cs-archive.uwaterloo.ca/ cs-archive/CS-2000-04/.

[BL98] Bonnie Berger and Tom Leighton. Protein folding in the hydrophobic-hydrophilic (HP) model is NP-complete. Journal of Computational Biology, 5(1):27-40, 1998.

[BM56] W. Blaschke and H. R. Müller. Ebene Kinematik. Verlag von R. Oldenbourg, München, 1956.

[BR79a] O. Bottema and B. Roth. Theoretical Kinematics. North-Holland Publishing Company, Amsterdam, 1979. Reprinted by Dover Publications, 1990.

[BR79b] O. Bottema and B. Roth. Theoretical kinematics, volume 24 of North-Holland Series in Applied Mathematics and Mechanics. North-Holland Publishing Co., Amsterdam, 1979.

[Bri96] R. Bricard. Sur une question de géométrie relative aux polyèdres. Nouv. Ann. Math., 15:331-334, 1896.

[BS90] A. V. Bushmelev and I. Kh. Sabitov. Configuration spaces of Bricard octahedra. Ukrain. Geom. Sb., 33:36-41, ii, 1990.

[Can87] John F. Canny. The Complexity of Robot Motion Planning. MIT Press, Cambridge, 1987.

[CD93] Hue Sun Chan and Ken A. Dill. The protein folding problem. Physics Today, pages 24-32, Feb. 1993.

[CDIO02] Jason H. Cantarella, Erik D. Demaine, Hayley N. Iben, and James F. O'Brien. An energy-driven approach to linkage unfolding. Manuscript, 2002. Abstract presented at the DIMACS Workshop on Computational Geometry. 
[CDR] Robert Connelly, Erik D. Demaine, and Günter Rote. Straightening polygonal arcs and convexifying polygonal cycles. Discrete Comput. Geom. To appear. Preliminary version appeared at FOCS 2000.

[CDR02] Robert Connelly, Erik D. Demaine, and Günter Rote. Infinitesimally locked selftouching linkages with applications to locked trees. In J. Calvo, K. Millett, and E. Rawdon, editors, Physical Knots: Knotting, Linking, and Folding of Geometric Objects in 3-space, pages 287-311. American Mathematical Society, 2002.

$\left[\mathrm{CGP}^{+}\right.$98] Pierluigi Crescenzi, Deborah Goldman, Christos Papadimitriou, Antonio Piccolboni, and Mihalis Yannakakis. On the complexity of protein folding. Journal of Computational Biology, 5(3), 1998.

[CH88] G. M. Crippen and T. F. Havel. Distance geometry and molecular conformation, volume 15 of Chemometrics Series. Research Studies Press Ltd., Chichester, 1988.

[Che02] Chih-Hsin Chen. Kinemato-geometrical methodology for analyzing curvature and torsion of trajectory curve and its applications. Mech. Mach. Theory, 37(1):35-47, 2002.

[CJ] Robert Connelly and B. Jaggi. Unpublished.

[CJ98] Jason Cantarella and Heather Johnston. Nontrivial embeddings of polygonal intervals and unknots in 3-space. J. Knot Theory Ramifications, 7(8):1027-1039, 1998.

$\left[\mathrm{CKM}^{+} 01\right]$ Jorge Alberto Calvo, Danny Krizanc, Pat Morin, Michael Soss, and Godfried Toussaint. Convexifying polygons with simple projections. Infor. Process. Lett., 80(2):8186, 2001.

[CO01] Roxana Cocan and Joseph O'Rourke. Polygonal chains cannot lock in 4D. Discrete Comput. Geom., 20(3):105-129, Nov. 2001.

[Con78] Robert Connelly. The rigidity of suspensions. J. Differential Geom., 13(3):399-408, 1978.

[Con79] Robert Connelly. The rigidity of polyhedral surfaces. Math. Mag., 52(5):275-283, 1979.

[CP91] C. R. Calladine and S. Pellegrino. First-order infinitesimal mechanisms. Internat. J. Solids Structures, 27(4):505-515, 1991.

[Cri92] Gordon M. Crippen. Exploring the conformation space of cycloalkanes by linearized embedding. J. Comput. Chem., 13(3):351-361, 1992.

[Dem00] Erik D. Demaine. Folding and unfolding linkages, paper, and polyhedra. In Proc. Japan Conf. Discrete Comput. Geom., volume 2098 of Lecture Notes in Comput. Sci., pages 113-124, Tokyo, Japan, Nov. 2000.

[Dem02] Erik D. Demaine. Folding and Unfolding. PhD thesis, Dept. of Computer Science, U. Waterloo, 2002.

[Dil90] Ken A. Dill. Dominant forces in protein folding. Biochemistry, 29(31):7133-7155, Aug. 1990.

[DLOS] Erik D. Demaine, Stefan Langerman, Joseph O'Rourke, and Jack Snoeyink. Interlocked open and closed linkages with few joints. Comput. Geom. Theory Appl. To appear. Preliminary version in CCCG 2001.

[DLOS02] Erik D. Demaine, Stefan Langerman, Joseph O'Rourke, and Jack Snoeyink. Interlocked open linkages with few joints. In Proc. 18th Sympos. Comput. Geom., pages 189-198, Barcelona, Spain, June 2002. 
[Erd35] Paul Erdős. Problem 3763. Amer. Math. Monthly, 42:627, 1935.

$\left[\mathrm{FHM}^{+} 01\right]$ Thomas Fevens, Antonio Hernandez, Antonio Mesa, Michael Soss, and Godfried Toussaint. Simple polygons that cannot be deflated. Beiträge Algebra Geom., 2001. To appear.

[GHM97] C. G. Gibson, C. A. Hobbs, and W. L. Marar. On versal unfoldings of singularities for general two-dimensional spatial motions. Acta Appl. Math., 47(2):221-242, 1997.

[GN86] C. G. Gibson and P. E. Newstead. On the geometry of the planar 4-bar mechanism. Acta Appl. Math., 7(2):113-135, 1986.

[Grü95] Branko Grünbaum. How to convexify a polygon. Geombinatorics, 5:24-30, July 1995.

[GZ01] Granko Grünbaum and Joseph Zaks. Convexification of polygons by flips and by flipturns. Discrete Math., 241(1-3):333-342, 2001.

[Har74] H. Hart. On certain conversions of motion. Messenger Math., T. IV:82-88, 1874.

[Hau91] Jean-Claude Hausmann. Sur la topologie des bras articulés. In Algebraic topology Poznań 1989, volume 1474 of Lecture Notes in Math., pages 146-159. Springer, Berlin, 1991.

[Hav91] Timothy F. Havel. Some examples of the use of distances as coordinates for Euclidean geometry. J. Symbolic Comput., 11(5-6):579-593, 1991. Invariant-theoretic algorithms in geometry (Minneapolis, MN, 1987).

[Hay98] Brian Hayes. Prototeins. American Scientist, 86:216-221, 1998.

[HI96] William E. Hart and Sorin Istrail. Fast protein folding in the hydrophobic-hydrophilic model within three-eighths of optimal. Journal of Computational Biology, 3(1):53-96, Spring 1996.

[HJW84] John Hopcroft, Deborah Joseph, and Sue Whitesides. Movement problems for 2dimensional linkages. SIAM J. Comput., 13(3):610-629, Aug. 1984.

[HJW85] John Hopcroft, Deborah Joseph, and Sue Whitesides. On the movement of robot arms in 2-dimensional bounded regions. SIAM J. Comput., 14(2):315-333, May 1985.

[Hun78] K. H. Hunt. Kinematic geometry of mechanisms. The Clarendon Press Oxford University Press, New York, 1978. The Oxford Engineering Science Series.

[Jag92] B. Jaggi. Pinktmengen mit vorgeschriebenen Distanzen und ihre Konfigurationsräurne. Inauguraldissertation, Universität Bern, 1992.

[JP85] Deborah A. Joseph and W. Harry Plantings. On the complexity of reachability and motion planning questions. In Proceedings of the 1st Annual Symposium on Computational geometry, pages 62-66, Baltimore, Maryland, 1985.

[JS99] D. Jordan and M. Steiner. Configuration spaces of mechanical linkages. Discrete Comput. Geom., 22(2):297-315, 1999.

[Kam99] Yasuhiko Kamiyama. Topology of equilateral polygon linkages in the Euclidean plane modulo isometry group. Osaka J. Math., 36(3):731-745, 1999.

[Kem76] A. B. Kempe. On a general method of describing plane curves of the $n^{\text {th }}$ degree by linkwork. Proceedings of the London Mathematical Society, 7:213-216, 1876.

[Kem77] A. B. Kempe. How to Draw a Straight Line: A Lecture on Linkages. Macmillan and co., London, 1877.

[Kin] Henry C. King. Configuration spaces of linkages in $\mathbb{R}^{n}$. arXiv:math.GT/9811138. http://arXiv.org/abs/math.GT/9811138. 
[Kin99] Henry C. King. Planar linkages and algebraic sets. Turkish J. Math., 23(1):33-56, 1999.

[KM95] Michael Kapovich and John Millson. On the moduli space of polygons in the Euclidean plane. J. Differential Geom., 42(1):133-164, 1995.

[KM02] Michael Kapovich and John J. Millson. Universality theorems for configuration spaces of planar linkages. Topology, 41(6):1051-1107, 2002.

[KT99] Yasuhiko Kamiyama and Michishige Tezuka. Topology and geometry of equilateral polygon linkages in the Euclidean plane. Quart. J. Math. Oxford Ser. (2), 50(200):463470, 1999.

[Leb67] Henri Lebesgue. Octaèdres articulés de Bricard. Enseignement Math. (2), 13:175-185 (1968), 1967.

[Ler00] Jean Lerbet. Some explicit relations in kinematics of mechanisms. Mech. Res. Comm., 27(6):621-630, 2000.

[Lip71] L. Lipkin. Dispositif articulé pour la transformation rigoureuse du mouvement circulaire en mouvement rectiligne. Revue Univers. des Mines et de la Métallurgie de Liége, 30(4):149-150, 1871.

[LW95] W. J. Lenhart and S. H. Whitesides. Reconfiguring closed polygonal chains in Euclidean $d$-space. Discrete Comput. Geom., 13(1):123-140, 1995.

[McC90] J. Michael McCarthy. An introduction to theoretical kinematics. MIT Press, Cambridge, MA, 1990.

[McC00] J. Michael McCarthy. Geometric design of linkages, volume 11 of Interdisciplinary Applied Mathematics. Springer-Verlag, New York, 2000.

[Mik01] S. N. Mikhalev. Some necessary metric conditions for the flexibility of suspensions. Vestnik Moskov. Univ. Ser. I Mat. Mekh., 3:15-21, 77, 2001.

[Mnë88] N. E. Mnëv. The universality theorems on the classification problem of configuration varieties and convex polytopes varieties. In Topology and geometry-Rohlin Seminar, volume 1346 of Lecture Notes in Math., pages 527-543. Springer, Berlin, 1988.

[MS00] Olivier Mermoud and Marcel Steiner. Visualisation of configuration spaces of polygonal linkages. J. Geom. Graph., 4(2):147-157, 2000.

[Nag39] Béla de Sz. Nagy. Solution to problem 3763. Amer. Math. Monthly, 46:176-177, Mar. 1939.

[New02] Alantha Newman. A new algorithm for protein folding in the HP model. In Proc. 13th ACM-SIAM Sympos. Discrete Algorithms, pages 876-884, San Francisco, Jan. 2002.

[O'R98] Joseph O'Rourke. Folding and unfolding in computational geometry. In Revised Papers from the Japan Conf. Discrete Comput. Geom., volume 1763 of Lecture Notes in Comput. Sci., pages 258-266, Tokyo, Japan, Dec. 1998.

[Pea73] A. Peaucellier. Note sur une question de géometrie de compas. Nouv. Ann. de Math., 2e serie, T. XII:71-73, 1873.

[Pot94] Helmut Pottmann. Kinematische Geometrie. In Geometrie und ihre Anwendungen, pages 141-175. Hanser, Munich, 1994.

[Rob75] S. Roberts. On three-bar motion in plane space. Proceedings of the London Mathematical Soceity, 7:14-23, November 1875. 
[SEO01] Michael Soss, Jeff Erickson, and Mark Overmars. On the difficulty of preprocessing for dihedral rotation queries. Manuscript in preparation, 2001.

[Sos01] Michael Soss. Geometric and Computational Aspects of Molecular Reconfiguration. PhD thesis, School of Computer Science, McGill University, 2001.

[ST00] Michael Soss and Godfried T. Toussaint. Geometric and computational aspects of polymer reconfiguration. Journal of Mathematical Chemistry, 27(4):303-318, 2000.

[Sta97] Hellmuth Stachel. Euclidean line geometry and kinematics in the 3-space. In Proceedings of the 4th International Congress of Geometry (Thessaloniki, 1996), pages 380-391. Giachoudis-Giapoulis, Thessaloniki, 1997.

[Sta99] Hellmuth Stachel. Higher order flexibility of octahedra. Period. Math. Hungar., 39(13):225-240, 1999. Discrete geometry and rigidity (Budapest, 1999).

[Str00] Ileana Streinu. A combinatorial approach to planar non-colliding robot arm motion planning. In Proc. 41st IEEE Sympos. Found. Comp. Sci., pages 443-453, Redondo Beach, California, Nov. 2000.

[Tou99] Godfried Toussaint. The Erdős-Nagy theorem and its ramifications. In Proc. 11th Canadian Conf. Comput. Geom., Vancouver, Canada, Aug. 1999. http://www.cs . ubc.ca/conferences/CCCG/elec_proc/fp19.ps.gz.

[Tou01] Godfried Toussaint. A new class of stuck unknots in pol 6 . Beiträge Algebra Geom., 42(2):1027-1039, 2001.

[TW84] W. Thurston and J. Weeks. The mathematics of three-dimensional manifolds. Scientific American, pages 108-120, July 1984.

[Weg93] Bernd Wegner. Partial inflation of closed polygons in the plane. Beiträge Algebra Geom., 34(1):77-85, 1993. 